\title{
Gamma Knife for Giant Hypothalamic Hamartomas-Not the Only Effective Tool in the Kit
}

\author{
P. Sarat Chandra ${ }^{1}$ (I) $\cdot$ Ramesh Doddamani $^{1} \cdot$ Manjari Tripathi $^{2}$
}

Received: 9 July 2021 / Accepted: 15 July 2021 / Published online: 1 September 2021

(c) Dr. K C Chaudhuri Foundation 2021

Hypothalamic hamartomas form a rare cause of drugresistant epilepsy (DRE) in children. Though commonly occurring in the hypothalamus, they may rarely occur in other areas like posterior fossa [1]. The most common presentation is gelastic epilepsy (61\%), followed by precocious puberty.

DRE in children must be treated as early as possible through surgery, otherwise it can have disastrous consequences on cognition, and can also increases the risk of death $[2,3]$. The only randomized trial involving children with drug-resistant epilepsy to compare medical and surgical therapy revealed a tenfold better seizure-free outcome for those who underwent early surgery as compared with those who underwent medical therapy only $[2,3]$.

Hence, once the diagnosis of hypothalamic hamartoma is made, surgery becomes the only option to be considered.

As enumerated by Tripathi et al. [4] various treatment options are available, the most known ones include open surgery, endoscopic ultrasound-guided disconnection, stereotactic radiosurgery, stereotactic radiofrequency thermocoagulation, and laser interstitial thermocoagulation therapy (LITT).

Open surgery has been more or less given up, as it involves open craniotomy and higher morbidity, especially in children. In addition, it approaches the hamartoma from the outer surface which has a higher risk of injury to the vascular supply to hypothalamus. Endoscopic ultrasound-guided disconnection involves the use of neuronavigation, which allows accurate guidance of the endoscope into the ventricle to the interface between the lesion and the brain. A disconnection is then performed using a bipolar and a

P. Sarat Chandra

saratpchandra@aiims.edu

1 Departments of Neurosurgery, All India Institute of Medical Sciences, New Delhi 110029, India

2 Department of Neurology, Epilepsy \& MEG Resource Facility, All India Institute of Medical Sciences, New Delhi, India biopsy forceps [5]. LITT is a recent development [6] which is performed in the MRI suite after the electrode has been placed in the hamartoma. The temperature and the extent of thermocoagulation can be monitored in real time; thus, the preoperative planning allows accurate and safe lesioning. However, the main disadvantage of this procedure includes limitation to a single trajectory, which makes it more suitable for small hamartomas and not giant hamartomas. The laser electrodes are also expensive (> 1000 USD per electrode), and its single use limits its utility in developing countries like India.

Tripathi et al. [4] have explored the utility of primary hypofractionated gamma knife for giant hypothalamic hamartomas in 3 cases. Of these, one had class I and two had class III outcome at a follow-up between 24-30 mo. Similar studies in literature also demonstrated class I outcome in up to $60 \%$ of the patients.

We have developed the technique of robotic guided stereotactic radio-frequency thermocoagulation for hypothalamic hamartomas [7, 8]. Till date, out of 25 patients treated using this technique, 5 had giant hamartomas; $4 / 5$ of these patients had class I outcome; 2 required more than 1 surgery. The main advantage of this procedure was the ability to use multiple trajectories, being minimally invasive (single suture, through twist drills), least morbidity and no blood loss. After having more than $20 \mathrm{y}$ of experience of treating hypothalamic hamartomas with various techniques, we have settled down with this procedure due to its efficacy and safety. The procedure is also quite economical costing only Rs 2000/(20 USD) per patient as the entire equipment is reusable.

To summarize, several new modalities are emerging. LITT is now being widely used in the US where the medical care is covered through insurance. Gamma knife may be promising, but long-term results need to be studied. The current study has involved only 3 patients; hence, a longer follow-up is needed. Also, one should take into caution that only $1 / 3$ patients had class I outcome. In our institute, we have found robotic guided stereotactic radiofrequency 
ablation to be very satisfactory with almost $90 \%$ class I results with negligible morbidity.

\section{Declarations}

Conflict of Interest None.

\section{References}

1. Chandra PS, Gulati S, Kalra V, et al. Fourth ventricular hamartoma presenting with status epilepticus treated with emergency surgery in an infant. Pediatr Neurosurg. 2011;47:217-22.

2. Chandra PS, Ramanujam B, Tripathi M. Surgery for drug-resistant epilepsy in children. N Engl J Med. 2018;378:399.

3. Dwivedi R, Ramanujam B, Chandra PS, et al. Surgery for drugresistant epilepsy in children. N Engl J Med. 2017;377:1639-47.

4. Tripathi M, Maskara P, Sankhyan N, et al. Safety and efficacy of primary hypofractionated gamma knife radiosurgery for giant hypothalamic hamartoma. Indian J Pediatr. 2021. https://doi.org/ 10.1007/s12098-020-03637-w.

5. Akai T, Okamoto K, Iizuka H, Kakinuma H, Nojima T. Treatments of hamartoma with neuroendoscopic surgery and stereotactic radiosurgery: a case report. Minim Invasive Neurosurg. 2002;45:235-9.

6. Burrows AM, Marsh WR, Worrell G, et al. Magnetic resonance imaging-guided laser interstitial thermal therapy for previously treated hypothalamic hamartomas. Neurosurg Focus. 2016;41:E8.

7. Doddamani RS, Tripathi M, Samala R, et al. Hypothalamic hamartoma and endocrinopathy: a neurosurgeon's perspective. Neurol India. 2020;68:S146-53.

8. Tandon V, Chandra PS, Doddamani RS, et al. Stereotactic radiofrequency thermocoagulation of hypothalamic hamartoma using robotic guidance (ROSA) coregistered with O-arm guidancepreliminary technical Note. World Neurosurg. 2018;112:267-74.

Publisher's Note Springer Nature remains neutral with regard to jurisdictional claims in published maps and institutional affiliations. 